\title{
A Rare Disease in the Differential Diagnosis of Acute Pancreatitis: Acute Brucellosis
}

\author{
Ilhami Berber ${ }^{1}$, Mehmet Ali Erkurt ${ }^{1}$, Funda Yetkin ${ }^{2}$, Serkan Unlu ${ }^{3}$, Sami Y1maz ${ }^{4}$, \\ Recep Bentli ${ }^{4}$ and Sezai Bazna ${ }^{4}$
}

\begin{abstract}
Some infectious organisms may give rise to acute pancreatitis; brucellosis, however, extremely rarely leads to acute pancreatitis. A 40-year-old man was diagnosed with acute pancreatitis, the etiology of which was determined to be acute brucellosis. The patient was discharged without complications approximately 15 days after the initiation of trimethoprim-sulfamethoxazole and doxycycline treatment. Brucella infections may rarely be complicated by acute pancreatitis. Thus, brucellosis should be remembered in the etiology of acute pancreatitis in regions such as Turkey, where Brucella infections are endemic.
\end{abstract}

Key words: acute pancreatitis, brucellosis, complication

(Intern Med 53: 2401-2404, 2014)

(DOI: 10.2169/internalmedicine.53.2510)

\section{Introduction}

Brucellosis is primarily a disease of herbivores caused by Brucella species. The transmission from other animals to humans is usually the result of direct contact of animal secretions with injured skin, ingestion of unpasteurized milk and dairy products, inhalation of infected aerosols, as well as conjunctival contact. In countries where the disease is endemic, as in Turkey, human transmission often occurs by consumption of unpasteurized dairy products (1).

Acute pancreatitis is defined as pancreatic inflammation. It is an important condition leading to considerable morbidity and mortality. Determining the etiology is one of the main components of the diagnostic process because the etiology can regulate the treatment and elimination of the etiologic agent can prevent recurrent attacks (2).

Brucellosis usually has a favorable prognosis. However, osteoarticular, meningeal and cardiovascular involvement can be encountered, leading to morbidity and mortality. Although rarely, brucellosis can also manifest itself as severe thrombocytopenia, capillary leak syndrome and acute pancreatitis (3-5).
We herein present a case of acute pancreatitis complicated with brucellosis which was improved following antibrucellosis treatment.

\section{Case Report}

A 40-year old Turkish farmer was admitted to our clinic with fever, muscle and abdominal pain and weakness. His blood pressure was $104 / 70 \mathrm{mmHg}$, body temperature $38.3^{\circ} \mathrm{C}$ and pulse rate $94 \mathrm{bpm}$. On physical examination, his sclera and skin were icteric and he had diffuse epigastric tenderness along with mild abdominal distension. However, he had no organomegaly or ascites. His physical examination was otherwise unremarkable. The hematological and biochemical results are as indicated (Table): the peripheral blood smear revealed $70 \%$ neutrophils, $25 \%$ lymphocytes, $5 \%$ monocytes and normally aggregating thrombocytes. The patient's chest film and electrocardiography were also within the normal limits. An abdominal ultrasonography was ordered, but the pancreas could not be properly visualized due to diffuse abdominal gas. A subsequent abdominal computed tomography (CT) revealed peripancreatic fluid and pancreatic edema (Fig. 1). The patient was diagnosed with acute pancreatitis

\footnotetext{
${ }^{1}$ Department of Hematology, Faculty of Medicine, Inonu University, Turkey, ${ }^{2}$ Department of Infection Disease, Faculty of Medicine, Inonu University, Turkey, ${ }^{3}$ Department of Radiology, Faculty of Medicine, Inonu University, Turkey and ${ }^{4}$ Department of Internal Medicine, Faculty of Medicine, Inonu University, Turkey

Received for publication January 20, 2014; Accepted for publication April 8, 2014

Correspondence to Dr. Ilhami Berber, drilhamiberber@hotmail.com
} 
Table. Laboratory Results of the Patient at the Time of Diagnosis

\begin{tabular}{lrr}
\hline Parameters & Result & Normal Range \\
\hline Glucose & 85 & $70-105 \mathrm{mg} / \mathrm{dL}$ \\
BUN & 23 & $8-20 \mathrm{mg} / \mathrm{dL}$ \\
Creatinine & 0.8 & $0.7-1.2 \mathrm{mg} / \mathrm{dL}$ \\
AST & 534 & $5-34 \mathrm{U} / \mathrm{L}$ \\
ALT & 219 & $0-55 \mathrm{U} / \mathrm{L}$ \\
Total Bilirubin & 15.3 & $0.2-1.2 \mathrm{mg} / \mathrm{dL}$ \\
D.Bilirubin & 13.2 & $0-0.5 \mathrm{mg} / \mathrm{dL}$ \\
Amilase & 1,123 & $25-125 \mathrm{U} / \mathrm{L}$ \\
Lipase & 2,822 & $8-78 \mathrm{U} / \mathrm{L}$ \\
Sodium & 135 & $136-145 \mathrm{mmol} / \mathrm{L}$ \\
Potassium & 4.2 & $3.5-5.1 \mathrm{mmol} / \mathrm{L}$ \\
Leucocyte count & 7,300 & $4.000-10.000 \mathrm{~mm}$ \\
Hemoglobin & 13 & $13-17 \mathrm{~g} / \mathrm{dL}$ \\
Thrombocyte count & 180.000 & $150.000-450.000 \mathrm{~mm}$ \\
C-Reactive Protein & 24 & $0-0.35 \mathrm{mg} / \mathrm{dL}$ \\
\hline
\end{tabular}

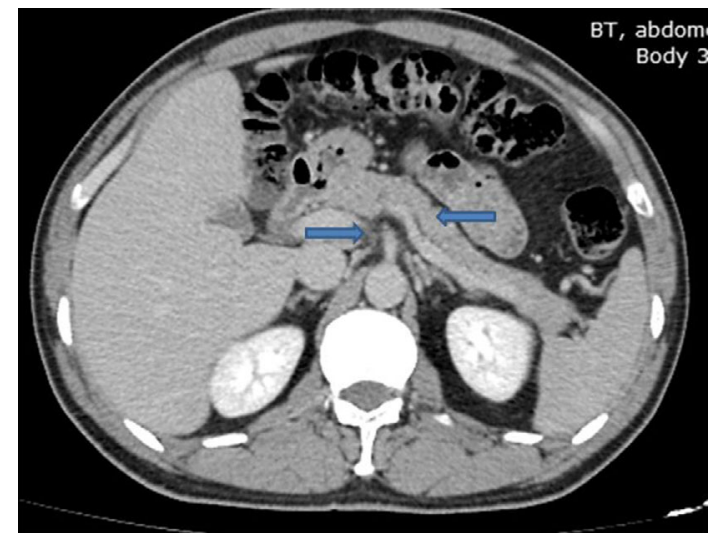

Figure 1. The right blue arrow shows minimal peripancreatic fluid and the left blue arrow shows minimal pancreatic duct dilatation. The head of the pancreas has an edematous appearance.

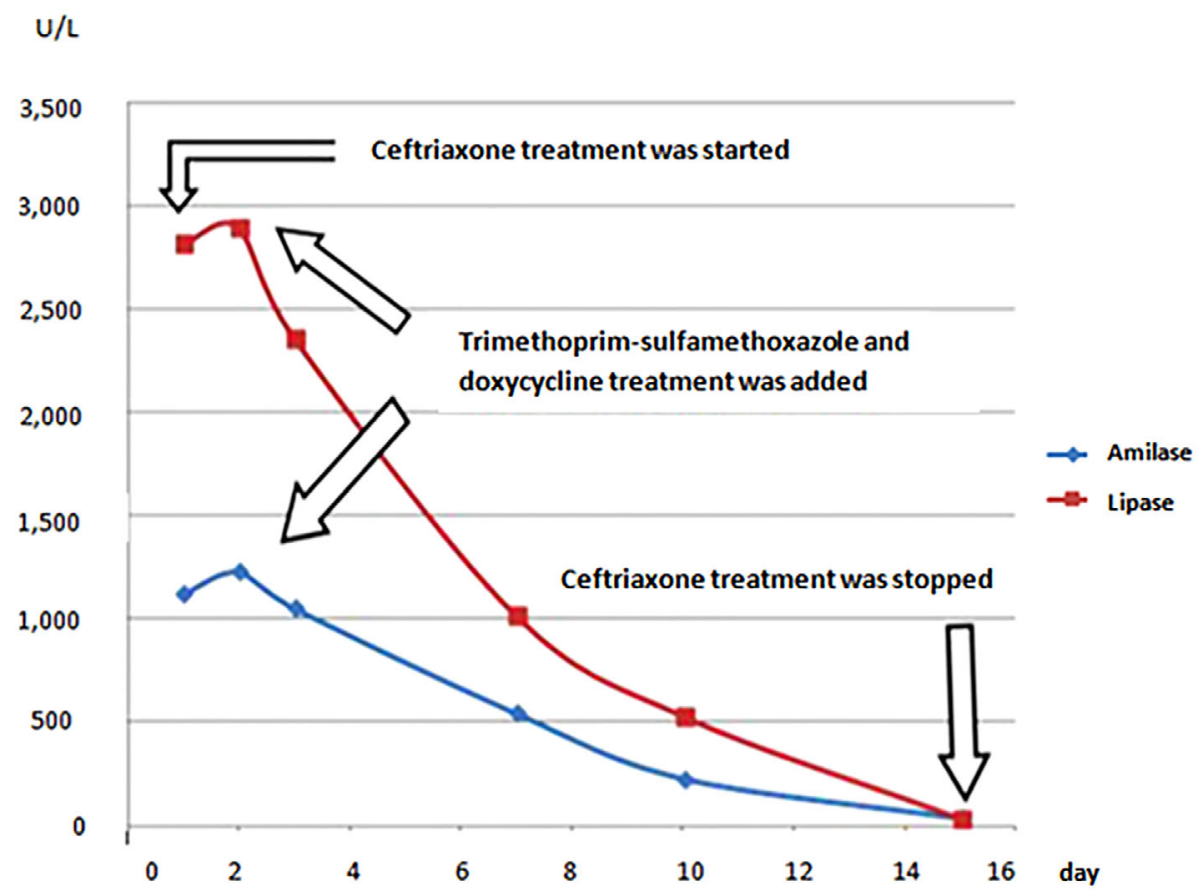

Figure 2. Decreasing amylase and lipase concentrations according to day

and was started on intravenous fluids, no oral feeding, esomeprazole (1×40 mg/day/intravenously), hyoscine-n-butyl bromide $(2 \times 20 \mathrm{mg} /$ day/intravenously) and ceftriaxone $(1 \mathrm{~g}$ bid IM). His past history did not note any previously similar attacks, alcohol addiction, cholelithiasis, parotitis or skin rashes, abdominal trauma or surgery or consumption of unpasteurized dairy products. His lipid profile was also normal. The testing for viral antibodies against cytomegalovirus, mumps, Epstein-Barr virus, herpes simplex viruses I and II, HIVI and II, Hepatitis A, B and C viruses and echoviruses were all negative. The serological tests directed at Mycoplasma, Legionella and toxoplasmosis were also negative. A purified protein derivative (PPD; Mantoux) skin test was also normal. Because the family members of the patient had a history of brucellosis and eating unpasteurized dairy products, he underwent tests specific for Brucella in- fection. The brucella lam agglutination test was positive, the Coombs antiserum test was $1 / 640$, and the Wright test was 1/320. A blood culture yielded Brucella melitensis proliferation. Upon this finding, trimethoprim-sulfamethoxazole (160/800 mg orally every 12 hours) and doxycycline (100 mg orally every 12 hours) were administered on the second day of his diagnosis with acute pancreatitis. His abdominal pain subsided three days later. He was started on oral feeding with a low-fat diet. On the 15th day of his admission to the clinic, his serum amylase and lipase levels returned to normal (Fig. 2). A follow-up abdominal CT showed regressed peripancreatic fluid. The ceftriaxone treatment was stopped, and the patient was discharged on trimethoprimsulfamethoxazole and doxycycline therapy. No sign of Brucella infection was present at the follow-up appointment two months later and the trimethoprim-sulfamethoxazole and 


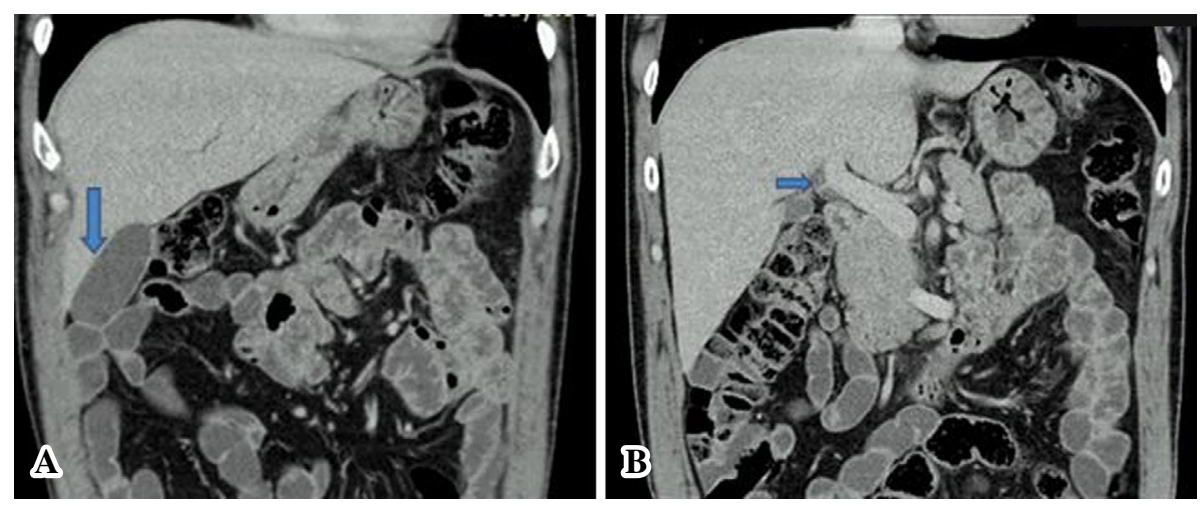

Figure 3. (A) The blue arrow on the coronal CT image shows a normal gallbladder without stones.

(B) The common bile duct is displayed at a normal size without gallstones (blue arrow).

doxycycline therapy was stopped. Furthermore, the patient was asymptomatic, had a normal serum amylase level and his serum agglutination for brucellosis was only $1 / 80$.

\section{Discussion}

Determining the etiology of acute pancreatitis is a crucial part of the diagnostic process. A wide spectrum of conditions can give rise to acute pancreatitis, including biliary stones, alcoholism, hypertriglyceridemia and endoscopic retrograde cholangiopancreatography. More rarely, bacterial, viral and parasitic infections can cause acute pancreatitis $(6,7)$. Among the causative bacterial pathogens are $M y$ coplasma pneumoniae, Salmonella, Shigella, Campylobacter, hemorrhagic Escherichia coli, Legionella and Leptospira which can trigger acute pancreatitis by secreting toxins that damage the pancreas $(6,8)$. Our patient had no history of biliary stones; the width of the ductus choledochus and thickness of the gallbladder wall were normal for passing stones (Fig. 3). He was a farmer, had a history of family brucellosis and eating unpasteurized dairy products, so these findings were suggestive of Brucella which may have travelled into the pancreas through the biliary system and blood of our patient.

In the 1930s and 1940s, pancreatic localization of Brucella was an autopsy finding that was rarely reported in patients with active brucellosis. Madkour and Karawi were the first to report a case of acute brucellar pancreatitis (9). AlAwadhi et al. reported the second case of acute pancreatitis associated with brucellosis (5). This report detailed the occurrence of acute pancreatitis with concurrent positive blood cultures for Brucella melitensis (5). Aleman et al. reported a case of acute pancreatitis during the course of brucellosis (10). Odeh et al. reported a similar case of acute pancreatitis associated with brucellosis (11). Papaioannides et al. reported a patient who presented with acute pancreatitis, fever, myalgia and other clinical symptoms. Brucella melitensis was cultured from his blood. All clinical manifestations gradually resolved with the administration of intramuscular streptomycin and oral doxycycline therapy (12). Turkulov et al. reported a study on the various clinical manifestations of brucellosis infection. They investigated 15 patients treated for Brucella infection at the Clinic for Infectious Diseases between 2004 and 2005. Three patients had clinical features of knee arthritis, two had bronchopneumonia, one had pancreatitis and one developed signs of acute kidney failure (13). Rombola et al. reported a patient who developed acute pancreatitis five days after the early termination of antibiotic therapy (12 days of doxycycline and rifampin). It was decided that the patient should start doxycycline $(200 \mathrm{mg} /$ day $)$ and streptomycin $(1 \mathrm{~g} /$ day $)$. After three days of therapy, there was a complete remission of abdominal pain, and the patient's amylase and lipase levels returned to normal 13 days after discharge (14). In Madkour's series of 500 patients with active brucellosis, acute pancreatitis occurred in only one patient (9). Colmenero et al. reported a prospective study of 530 patients with brucellosis in Spain. In that study, the most common complications were, in descending order, osteoarticular $(n=113 ; 66 \%)$, genitourinary $(\mathrm{n}=18 ; 5.1 \%$ of males), hepatic $(2.5 \%)$, neurologic $(1.7 \%)$ and cardiac $(1.5 \%)$ complications. Nine (1.7\%) patients had multiple complications. However, no pancreatic involvement was seen, showing the rarity of pancreatic involvement during the course of a Brucella infection (15). Cholecystitis, colitis, and ileitis cases dependent on brucellosis have also been reported (16). Brucellainduced pancreatitis has features similar to other types of pancreatitis caused by other reasons (9) and brucellosis can mimic every kind of clinical presentation. Therefore, its diagnosis poses a challenge because of the spectrum of clinical presentation. Active infection is determined by a $1 / 160$ or higher standard agglutination test result in most cases (17).

Therapy for patients with Brucella-induced acute pancreatitis consists of supportive treatment with intravenous fluids, gastric decompression with nasogastric tube suctions, and analgesics and antimicrobial therapy directed against active Brucella infection (2). Literature data suggest that the infection favorably responds to appropriate therapy. Hence, our patient also fully improved following initiation of antimicrobial therapy consisting of trimethoprim-sulfamethoxazole and doxycycline. In summary, Brucella-induced acute pan- 
creatitis should be considered in geographical regions, such as Turkey, where Brucella infections are endemic. The treatment of acute pancreatitis may be as simple as administering antimicrobial therapy to treat the Brucella infection.

The authors state that they have no Conflict of Interest (COI).

\section{References}

1. Ulu-Kilic A, Metan G, Alp E. Clinical presentations and diagnosis of brucellosis. Recent Pat Antiinfect Drug Discov 8: 34-41, 2013.

2. Wu BU, Banks PA. Clinical management of patients with acute pancreatitis. Gastroenterology 144: 1272-1281, 2013.

3. Erkurt MA, Sari I, Gül HC, Coskun O, Eyigün CP, Beyan C. The first documented case of brucellosis manifested with pancytopenia and capillary leak syndrome. Intern Med 47: 863-865, 2008.

4. Elmas Dal S, Ersoy Y, Ali Erkurt M, Yetkin F, Kuzucu C, Akdogan O. An uncommon case of acute brucellosis presenting with severe thrombocytopenia. Intern Med 51: 3291-3293, 2012.

5. Al-Awadhi NZ, Ashkenani F, Khalaf ES. Acute pancreatitis associated with brucellosis. Am J Gastroenterol 84: 1570-1574, 1989.

6. Topazian M, Gorelick FS. Acute pancreatitis. In: Textbook of Gastroenterology. 4th ed. Yamada T, Alpers DH, Kaplowitz N, Laine L, Dwyang C, Powell DW, Eds. Lippincott Williams and Wilkins, Philadelphia, USA, 2003: 2026-2061.

7. Parenti DM, Steinberg W, Kang P. Infectious causes of acute pancreatitis. Pancreas 13: 356-371, 1996.

8. Leinikki P, Pantzar P, Tykka H. Antibody response in patients with acute pancreatitis to mycoplasma pneumoniae. Scand J Gastroenterol 8: 631-635, 1973.

9. Madkour Monir M. Gastrointestinal brucellosis. In: Madkour's Brucellosis. 2nd ed. Madkour Monir M, Ed. Springer-Verlag, Berlin, Germany, 2001: 150-158.

10. Aleman MC, Alegre J, Marques A, Surinach JM, Fernadez de Sevilla T. Acute pancreatitis in the course of brucellosis. An Med Interna 11: 311, 1994.

11. Odeh M, Oliven A. Acute pancreatitis associated with brucellosis. J Gastroenterol Hepatol 10: 691-692, 1995.

12. Papaioannides D, Korantzopoulos P, Sinapidis D, Charalabopoulos $\mathrm{K}$, Akritidis N. Acute pancreatitis associated with brucellosis. JOP 7: 62-65, 2006.

13. Turkulov V, Madle-Samardzija N, Canak G, Gavrancić C, Vukadinov J, Doder R. Various clinical manifestations of brucellosis infection. Med Pregl 61: 517-520, 2008.

14. Rombola F, Bertuccio SN. Acute pancreatitis after early termination of brucellosis antibiotic therapy. J Gastrointestin Liver Dis 17: 234, 2008.

15. Colmenero JD, Reguera JM, Martos F, et al. Complications associated with Brucella melitensis infection: a study of 530 cases. Medicine (Baltimore) 75: 195-211, 1996.

16. Caballeria F, Masso RM, Arago JV, Sanchis A. Ascites as the first manifestation of Brucella Granulomatous hepatitis. J Hepatol 15: 415-416, 1992.

17. Young EJ. Brucella species. In: Mandell, Douglas, and Bennett's Principles and Practice of Infectious Diseases. 6th ed. Mandell GL, Bennet JE, Dolin R, Eds. Elsevier Churchill Livingstone, Philadelphia, USA, 2005: 2669-2674.

(C) 2014 The Japanese Society of Internal Medicine http://www.naika.or.jp/imonline/index.html 